\title{
Peer victimisation and depressive symptoms: can specific coping strategies buffer the negative impact of cybervictimisation?
}

\author{
Katja Machmutow ${ }^{\mathrm{a}}$, Sonja Perren $^{\mathrm{a} *}$, Fabio Sticca $^{\mathrm{a}}$ and Françoise D. Alsaker ${ }^{\mathrm{b}}$ \\ aJacobs Center for Productive Youth Development, University of Zurich, Zurich, Switzerland; \\ ${ }^{b}$ Department of Psychology, University of Bern, Berne, Switzerland
}

\begin{abstract}
This longitudinal study investigated whether cybervictimisation is an additional risk factor for depressive symptoms over and beyond traditional victimisation in adolescents. Furthermore, it explored whether certain coping strategies moderate the impact of cybervictimisation on depressive symptoms. A total of 765 Swiss seventh graders (mean age at time-point $1(t 1)=13.18$ years) reported on the frequency of traditional and cybervictimisation, and of depressive symptoms twice in six months. At time-point $2(t 2)$ students also completed a questionnaire on coping strategies in response to a hypothetical cyberbullying scenario. Analyses showed that both traditional and cybervictimisation were associated with higher levels of depressive symptoms. Cybervictimisation also predicted increases in depressive symptoms over time. Regarding coping strategies, it was found that helpless reactions were positively associated with depressive symptoms. Moreover, support seeking from peers and family showed a significant buffering effect: cybervictims who recommended seeking close support showed lower levels of depressive symptoms at $t 2$. In contrast, cybervictims recommending assertive coping strategies showed higher levels of depressive symptoms at $t 2$.
\end{abstract}

Keywords: cyberbullying; cybervictimisation; depressive symptoms; coping; support; assertiveness; longitudinal

\section{Introduction}

It is well established that peer victimisation has negative short- and long-term consequences for children's and adolescents' mental health. Depressive symptoms are amongst the most prominent negative consequences of peer victimisation (Desjardins and Leadbeater 2011; Pouwelse et al. 2011). Past research focused mainly on the influence of victimisation on emotional well-being, but during recent years the inverse relationship has also gained attention: depressive symptoms have also been considered as a vulnerability factor for victimisation (Sweeting et al. 2006). Therefore, a reciprocal relationship may exist between victimisation experiences and depressive symptoms.

Cross-sectional studies suggest that being victimised in cyberspace is also associated with psychological difficulties (Mason 2008), stronger negative feelings, fear and feelings of helplessness (Spears et al. 2009), and also with depression (Ybarra 2004). Owing to the conceptual and empirical overlap between traditional and cybervictimisation (e.g. Perren and Gutzwiller-Helfenfinger 2012), the question arises whether cybervictimisation leads to

\footnotetext{
${ }^{*}$ Corresponding author. Email: perren@jacobscenter.uzh.ch
} 
similar consequences as traditional victimisation, and whether it represents an additional risk factor when traditional victimisation is controlled for. Several cross-sectional studies have shown that cybervictimisation is an additional risk factor for depressive symptoms (Gradinger, Strohmeier, and Spiel 2009; Perren et al. 2010; Wang, Nansel, and Iannotti 2011). Mitchell, Ybarra, and Finkelhor (2007) reported even higher levels of depressive symptoms in cybervictims than in traditional victims.

\section{Coping strategies in relation to cybervictimisation}

Coping, defined as the ability to manage stress and related emotions, is relevant for the sustainment of emotional and psychological well-being in the presence of adversity (Lazarus 2006). Coping strategies may therefore buffer the negative impact of cybervictimisation on depressive symptoms. Several types of coping strategies have been investigated in relation to experiences of cybervictimisation: supportive strategies (e.g. seeking social support from adults, teachers, friends or external institutions), reactions towards cyberbullies (e.g. retaliation, confrontation), technical strategies (e.g. report abuse buttons, blocking the sender), and avoiding and emotion-focused strategies (e.g. doing nothing, ignoring, helplessness).

\section{Support seeking}

Many students recommend asking their parents for help in case of a cybervictimisation incident (Aricak et al. 2008; Bhat 2008; Slonje and Smith 2008; Smith et al. 2008; Stacey 2009; Topcu, Erdur-Baker, and Capa-Aydin 2008). Other adolescents recommend not consulting adults because they fear losing enjoyable privileges (e.g. having and using mobile phones and their own Internet access), and because they expect that their parents would simply recommend ignoring the situation or that they would not be able to help them as they are not that familiar with cyberspace (Hoff and Mitchell 2009; Kowalski, Limber and Agatston 2008; Mishna, Saini, and Solomon 2009; Smith et al. 2008; Stacey 2009). In fact, Slonje and Smith (2008) suppose that adults are less aware of cybervictimisation as a problem. Therefore, parents tend to underestimate the seriousness of the problem and may be unable to give adequate support to their child. Self-reliance is another reason not to report cybervictimisation experiences to adults. In a web-based survey of 12-17-year-olds, most of whom had experienced at least one cybervictimisation incident in the year preceding the survey, Juvonen and Gross (2008) found that $90 \%$ of the victims did not tell their parents about their experiences and $50 \%$ of them justified it with 'I need to learn to deal with it myself'.

Although cyberbullying also happens outside the school context, victims often know their perpetrators from school (Smith and Slonje 2010), and teachers and the school, as a functional system, should not overlook or belittle cybervictimisation. Therefore, assistance from teachers and principals is implicitly necessary. However, students have a more negative and critical attitude to teachers' support: a large percentage of them consider telling a teacher or the school principal to be rather ineffective (Aricak et al. 2008; DiBasilio 2008; Mishna, Saini, and Solomon 2009; Mitchell, Finkelhor, and Wolak 2003; Price and Dalgleish 2010). Hoff and Mitchell (2009) found that although 17\% of students reported to a teacher after a cybervictimisation incident, in $70 \%$ of the cases the school did not react to it. Asking for help from peers is commonly used and recommended (Aricak et al. 2008; Bhat 2008; DiBasilio 2008; Stacey 2009; Topcu, Erdur-Baker, and Capa-Aydin 2008), although prevalence rates vary to a large extent. Slonje and Smith (2008) found that cybervictims were more likely to contact friends than other persons. At the same time, 
friends were less likely to consider cybervictimisation as relevant. Nonetheless, focusing on the perceived successfulness of coping strategies, Price and Dalgleish (2010) reported Australian cybervictims to consider 'telling a friend' as the most helpful strategy.

\section{Reactions towards the bully}

Confronting the bully is commonly reported by adolescents if the victim knows the bully or is able to contact her or him (Aricak et al. 2008; DiBasilio 2008; Stacey 2009). Although this strategy is often mentioned by those who experience victimisation, it has proved to be less helpful in retrospect (Price and Dalgleish 2010). Students consider retaliation as a less constructive way of contacting the perpetrator. When asking students what they had done to stop cybervictimisation, Hoff and Mitchell (2009) reported answers containing active and physically retaliatory behaviour, especially in boys. The assumption that bullying back through cyberspace is easier and therefore more often used than a face-to-face contact was not confirmed by Juvonen and Gross (2008): $60 \%$ of cybervictims defended against the bully in school using traditional means, whereas only $12 \%$ retaliated in cyberspace and $28 \%$ used both traditional and cyber retaliation.

\section{Technical solutions}

Cyberspace specific coping strategies such as deleting or blocking threatening messages are generally used and considered to be helpful (Aricak et al. 2008; Hinduja and Patchin 2007; Juvonen and Gross 2008; Kowalski, Limber, and Agatston 2008; Smith et al. 2008; Stacey 2009). Price and Dalgleish (2010) found that blocking was the most used technical strategy and was also considered the most helpful online action exerted by the self-identified cybervictims. Technical solutions are often reported along with preventive strategies used by parents, such as banning websites, and age-appropriate limits for the use of the computer and the Internet (Kowalski, Limber, and Agatston 2008).

\section{Avoidant and emotion-focused strategies}

According to coping theory (Roth and Cohen 1986), avoidance is a likely coping strategy in situations that are not under control of the affected person. Patchin and Hinduja (2006) reported $25 \%$ of victims doing nothing after a cybervictimisation incident. Dehue, Bolman, and Vollink (2008) found that $7.2 \%$ of students reported that with online victimisation they would usually pretend to ignore it, and $6.9 \%$ reported that they would ignore it. Chi and Frydenberg (2009) investigated the effects of a 10-week coping programme designed to reduce the negative impact of cybervictimisation. After completion of the programme the use of non-productive reactions, such as ignoring the problem, self-blaming or not coping, decreased and the level of emotional well-being increased. Tokunaga (2010) suggested that ignoring can be a very effective strategy following a single incident but that other (more active) coping strategies are more effective when the frequency and severity of episodes increase.

In sum, a range of coping strategies has been investigated in relation to cybervictimisation. However, most of the studies investigated the use (and not the success) of coping strategies in real cybervictims or in relation to hypothetical cybervictimisation. A few studies asked participants whether they think a certain coping strategy is successful (see also Perren et al. 2012). However, no study has yet investigated whether specific coping strategies buffer the negative impact of cybervictimisation on victims' emotional well-being. 


\section{Coping strategies and emotional well-being}

Two main dimensions are commonly differentiated in coping theory: emotion- versus problem-oriented coping strategies (Eschenbeck 2010). Some researchers see a problem in this subdivision because some coping strategies could be defined as both problem and emotion focused (e.g. support seeking). Consequently, a categorisation of strategies is not an ideal methodological approach (Skinner et al. 2003; Tenenbaum et al. 2011). As outlined above, current research on coping with cyberbullying has adopted a more descriptive approach and differentiates between specific behavioural and emotional reactions.

From a theoretical perspective we expect strong relationships between the use of specific coping strategies and an individual's emotional well-being (Lazarus 2006). Empirical studies show that emotion-focused coping is associated with distressing emotions, whereas problem-focused strategies are seen in association with increased emotional regulation and positive affect (Hampel, Manhal, and Hayer 2009; Yamasaki and Uchida 2006). Avoidant coping strategies are negatively associated with emotional well-being (e.g. Seiffge-Krenke and Klessinger 2000).

In studies on traditional victimisation, it has been supposed that avoidance is linked to negative outcomes such as maladjustment, loneliness, anxiety (Kochenderfer-Ladd and Skinner 2002) and other mental health problems (Ebata and Moos 1991). Lodge and Feldman (2007) found that avoidant coping partially mediated the association between appearance-related bullying problems and self-esteem among young adolescents. Kochenderfer-Ladd (2004) showed that students ignoring the victimisation (cognitive distancing) showed an increased risk for later harassment and were therefore indirectly at risk for a higher level of internalising symptoms. By investigating the relationship between victimisation and depressive symptoms, Singh and Bussey (2010) found mediating effects of how students, when imagining a hypothetical cybervictimisation incident, would rate their self-efficacy to use different coping strategies. Students being more victimised showed decreased self-efficacy scores regarding the resistance to debilitating thoughts and the ability to engage in enabling thoughts. These lowered scores were related to a higher level of depressive symptoms.

If victimisation is seen as a stressor affecting psychological well-being, the question arises as to whether specific coping strategies can buffer the negative outcomes of this association. Some studies aimed to test the so-called 'buffering hypothesis of social support' (e.g. Jackson 1992): there is some evidence that different forms of support minimise the negative impact of victimisation on psychological well-being (Davidson and Demaray 2007; Flouri and Buchanan 2002; Holt and Espelage 2007; KochenderferLadd and Skinner 2002). In contrast, Rigby (2000) and Pouwelse and colleagues (2011) found no evidence for the buffering hypothesis of social support, and Dooley and colleagues (2010) could not find associations between help-seeking behaviour and emotional symptoms.

All of the studies mentioned above focused on traditional victimisation when investigating mediating or moderating effects of coping strategies on the association between victimisation and psychological well-being. Yet, empirical evidence of the role of coping strategies on the association between cybervictimisation and depressive symptoms is missing, especially in a longitudinal context. The present study fills this gap by investigating moderating effects of different coping strategies in the association between cybervictimisation and depressive symptoms, using a longitudinal approach. 


\section{Research questions}

The first aim of this study was to investigate whether cybervictimisation is a longitudinal risk factor for depressive symptoms. We hypothesised that cybervictimisation predicts higher levels of depressive symptoms, even when controlling for traditional victimisation. The second aim was to explore the impact of specific coping strategies in relation to cybervictimisation on adolescents' depressive symptoms. We hypothesised that (1) support seeking (close support and distant advice) is associated with lower levels of depressive symptoms; and (2) avoidant and helpless reactions are associated with higher levels of depressive symptoms. Lastly, we explored whether specific coping strategies buffer the relationship between cybervictimisation and depressive symptoms. We hypothesised that support seeking buffers the negative impact of cybervictimisation.

\section{Method}

\section{Procedure}

Data are taken from the first (November/December 2010) and the second (May 2011) waves of data assessment in an ongoing longitudinal study carried out in Switzerland (netTEEN). Twelve randomly selected Swiss schools (43 classes) participated in the study. The assessments were conducted in three different cantons of Switzerland. The local languages of the schools were German (eight schools) and Italian (four schools). For the Italian-speaking schools questionnaires were translated from German into Italian by two bilingual native speakers.

All parents were informed about the study and invited to inform the respective teachers if they did not want their children to participate. A self-report questionnaire was administered in classrooms on netbooks. The questionnaire was administered by two research assistants. Students absent during the classroom assessment were asked to complete an online version of the questionnaire.

\section{Sample}

A total of 835 seventh graders participated in the first assessment $(t 1)$ and 820 students also participated in the second assessment $(t 2)$. Attrition was mainly due to adolescents no longer attending the school. Parents of four adolescents refused their children's participation. Because of time constraints, the coping measure was only completed by 765 students. Only these 765 students are included in the analysis $(52.1 \%$ female, age at $t 1$ : mean $=$ 13.18 years, $\mathrm{SD}=0.63$ ).

\section{Instruments}

Students reported on the frequency of cybervictimisation and traditional victimisation $(t 1$ and $t 2)$ and depressive symptoms ( $t 1$ and $t 2)$. The coping questionnaire was only completed at $t 2$.

\section{Victimisation}

Cybervictimisation was assessed with a self-developed six-item scale (Sticca et al. in press). Participants rated how often they had suffered from specific cyberbullying behaviours in the past four months. Possible responses ranged from 1 (never) to 5 (almost 
daily). The dimension of publicity (private, semi-public, public) was systematically varied in the items. The first two items (private) described an interaction involving only the bully and the victim (e.g. 'Did someone sent you nasty or threatening messages, i.e. text messages, MSN, Facebook, Netlog, etc.'). In the next two items (semi-public) the content (message, picture or video) would have been sent to groups of people (e.g. 'Are there adolescents sending nasty or embarrassing messages about you to other colleagues?'). In the last two items (public) the content would have been posted on the Internet (e.g. 'Are there adolescents publishing nasty or embarrassing pictures or videos on the internet?'). Item scores were averaged to gain an overall score of cybervictimisation $\left(\alpha \_t 1 / t 2=0.62 / 0.78\right)$, with higher scores indicating being more frequently victimised. Owing to its high skewness at the upper end of the scale, cybervictimisation was log transformed.

Involvement in traditional victimisation was assessed using an adapted version of a well-validated scale (Alsaker 2003; Alsaker and Brunner 1999). The items were introduced through a general definition of what bullying is. The scale consists of six items encompassing a set of different aggressive behaviours (e.g. being laughed at, insulted, excluded or hit by someone). Participants were asked how often they had suffered these behaviours in the past four months. Participants rated each item from 1 (never) to 5 (almost daily). Item scores were averaged to gain an overall score of traditional victimisation $\left(\alpha \_t 1 / t 2=0.76 / 0.81\right)$, with higher scores indicating being more frequently victimised. Owing to its high skewness at the upper end of the scale, traditional victimisation was log transformed.

\section{Depressive symptoms}

Students completed an eight-item scale addressing depressive symptoms at both assessment points. The scale has been validated in a longitudinal study (Alsaker 1992; Holsen, Kraft, and Vitterso 2000), and taps the following constructs: sad/depressed feelings (e.g. 'Sometimes I am just so depressed that I feel like staying in bed for the whole day'), lack of positive feeling, lack of motivation/energy, and worthlessness of life. Participants rated how much they agree with statements referring to the past four months. Possible responses ranged from 1 (not true) to 4 (true). Scores were averaged to obtain a single score for depressive symptoms $\left(\alpha_{-} t 1 / t 2=0.86 / 0.88\right)$. Higher scores indicate higher levels of depressive symptoms.

\section{Coping strategies}

Based on the results of a qualitative pilot study, a 14-item scale on coping strategies in relation to cybervictimisation was developed. The items were built based on adolescents' open answers regarding the perceived success of coping strategies in relation to cyberbullying (Machmutow and Perren 2011b). Students were given a hypothetical cyberbullying scenario (e.g. an embarrassing video was published on YouTube) and a list of 14 coping strategies was provided; they were asked what the hypothetical victim should do in this specific situation and rated each strategy on a scale ranging from 1 (definitely not) to 4 (definitely).

Each student was randomly given one cyberbullying scenario followed by the 14 items. The scenarios varied in publicity and severity of the incident as well as in the gender and victimisation experiences of the victim. The randomisation of the scenarios was applied to investigate the impact of situational variables on students' coping behaviour 
(Machmutow and Perren 2011a). The analyses presented here were statistically controlled for the situational variations of the scenarios.

Running a factor analysis, three distinct subscales (distant advice, assertiveness, helplessness) were found, together with two further subscales (close support, retaliation), which were examined separately (items tapping close support correlated with all subscales and retaliation was measured only with one item). One item ("not to take something to heart') turned out to be understood ambiguously and was eliminated from the following analyses.

With regard to support seeking, we specified two subscales: distant advice, e.g. attending an advice centre $(\alpha=0.67)$, and close support, e.g. talking to friends $(\alpha=0.65)$. Whereas distant advice implies the more informational and instrumental aspects of relief, close support targets the more emotional way of getting help from people (Cohen and Wills 1985). The subscale 'assertiveness', e.g. finding and contacting the bully ( $\alpha=0.49$ ), includes different means to defend oneself without causing harm to others (confronting the bully and using technical means). In contrast, retaliation (single item) is understood as a reaction through counter-aggression and revenge (Camodeca and Goossens 2005). Avoidant and emotion-focused coping strategies were combined in the subscale 'helplessness', e.g. self-blaming for the incident $(\alpha=0.36)$. This scale consists of three items referring to the attributions one can use when confronted with problematic situations (Kelley and Michela 1980): internal causes (e.g. self-blame), global causes (withdrawing, e.g. because bullying is everywhere) and stable causes (ignoring, e.g. because bullying is uncontrollable and therefore there is nothing you can do against it). More information or a copy of the (currently revised) coping questionnaire is available from the authors.

\section{Results}

\section{Descriptive statistics}

\section{Main effects of time, gender and age}

Means and gender differences of all study variables are shown in Table 1. Regarding differences between the two time-points of assessment, participants showed a decrease in traditional victimisation $(t(726)=3.79, p<0.001)$. The level of victimisation in cyberspace as well as the level of depressive symptoms did not differ between the two timepoints. Cyber and traditional victimisation were moderately stable. Depressive symptoms showed rather high stability. Girls reported higher levels of cybervictimisation $(t 1)$ and higher levels of depressive symptoms (both time-points). Girls more frequently recommended assertiveness and close support, and less frequently recommended retaliation as a coping strategy than boys.

\section{Victimisation, depressive symptoms and coping strategies}

Bivariate associations between all study variables are shown in Table 2. Cyber- and traditional victimisation were positively associated with each other, as well as with depressive symptoms at $t 1$ and $t 2$. Higher levels of retaliation and helplessness and lower levels of close support were associated with a higher level of depressive symptoms ( $t 1$ and $t 2$ ). Traditional victimisation $(t 2)$ was positively associated with higher levels of assertiveness and distant advice. Cybervictimisation $(t 2)$ was positively associated with retaliation and negatively with close support. 
Table 1. Means (standard deviations in parentheses) of all study variables $(n=345-388)$.

\begin{tabular}{|c|c|c|c|c|c|}
\hline & \multicolumn{2}{|c|}{$t 1$} & \multicolumn{2}{|c|}{$t 2$} & \multirow[b]{2}{*}{ Gender differences } \\
\hline & Female & Male & Female & Male & \\
\hline $\begin{array}{l}\text { Traditional } \\
\text { victimisation }\end{array}$ & $1.33(0.42)$ & $1.37(0.48)$ & $1.28(0.40)$ & $1.31(0.46)$ & ns \\
\hline Cybervictimisation & $1.10(0.26)$ & $1.06(0.19)$ & $1.08(0.19)$ & $1.06(0.25)$ & $\begin{array}{l}t 1: F(1,729)=6.05 \\
\quad p<0.05 \\
t 2: \mathrm{ns}\end{array}$ \\
\hline $\begin{array}{l}\text { Depressive } \\
\text { symptoms }\end{array}$ & $1.76(0.69)$ & $1.55(0.62)$ & $1.70(0.66)$ & $1.55(0.67)$ & $\begin{array}{l}t 1: F(1,719)=19.57 \\
\quad p<0.001 \\
t 2: F(1,732)=9.55 \\
\quad p<0.01\end{array}$ \\
\hline Distant advice & - & - & $2.57(0.77)$ & $2.60(0.85)$ & $\mathrm{ns}$ \\
\hline Close support & - & - & $3.23(0.60)$ & $2.97(0.84)$ & $\begin{array}{l}F(1,740)=24.08 \\
\quad p<0.001\end{array}$ \\
\hline Assertiveness & - & - & $3.05(0.61)$ & $2.95(0.73)$ & $\begin{array}{l}F(1,737)=4.36 \\
\quad p<0.05\end{array}$ \\
\hline Helplessness & - & - & $1.67(0.58)$ & $1.74(0.65)$ & ns \\
\hline Retaliation & - & - & $2.03(0.94)$ & $2.42(1.13)$ & $\begin{array}{l}F(1,725)=26.14 \\
\quad p<0.001\end{array}$ \\
\hline
\end{tabular}

Note: ns: not significant.

\section{Multivariate analyses}

Cross-sectional and longitudinal generalised linear models (GLMs) were used to investigate the impact of traditional and cybervictimisation on depressive symptoms. In a last step, we analysed whether coping strategies moderate the effects of cybervictimisation (interactions). As the coping questionnaire contained cyber-specific scenarios, we were only interested in a possible moderating effect of coping strategies on the association between cybervictimisation and depressive symptoms; models with interactions concerning traditional victimisation were not calculated.

\section{Predictors of depressive symptoms}

Cross-sectional associations. In a first step, age, gender and both forms of victimisation $(t 1)$ were included in a model to investigate their predictive value for depressive symptoms at $t 1$. Gender and both forms of victimisation yielded a significant effect. Being female (Wald $\chi^{2}(1,791)=21.42, B=0.20, p=0.001, \omega=0.16$ ), higher levels of traditional victimisation (Wald $\chi^{2}(1,791)=39.35, B=1.30, p=0.001, \omega=0.22$ ) and higher levels of cybervictimisation (Wald $\chi^{2}(1,791)=24.39, B=1.85, p=0.001, \omega=0.18$ ) predicted higher levels of depressive symptoms.

Longitudinal associations. For the longitudinal analyses, two models were computed: model 1 investigated the impact of victimisation at $t 1$ on depressive symptoms at $t 2$; in model 2 we controlled for depressive symptoms at $t 1$ and thus investigated the impact of victimisation on changes in depressive symptoms over time (Table 3). Age, gender, traditional and cybervictimisation ( $t 1$ and $t 2$ ), and coping strategies were used as independent variables. Gender and both types of victimisation at both time-points yielded significant main effects. Being female and higher frequency of victimisation were predictive of a 
Table 2. Bivariate associations between all study variables $(n=698-820)$.

\begin{tabular}{|c|c|c|c|c|c|c|c|c|c|c|}
\hline & 2 & 3 & 4 & 5 & 6 & 7 & 8 & 9 & 10 & 11 \\
\hline 1. Traditional victimisation $(t 1)$ & $0.43^{* *}$ & $0.27^{* *}$ & $0.50^{* * *}$ & $0.22^{* *}$ & $0.25^{* * *}$ & -0.04 & -0.03 & -0.01 & 0.04 & 0.00 \\
\hline 2. Cybervictimisation $(t 1)$ & 1 & $0.26^{* *}$ & $0.27^{* *}$ & $0.22^{* *}$ & $0.19^{* *}$ & 0.01 & -0.05 & -0.01 & -0.02 & 0.02 \\
\hline 3. Depressive symptoms $(t 1)$ & & 1 & $0.22^{* *}$ & $0.17^{* *}$ & $0.56^{* * *}$ & -0.06 & $-0.10^{* *}$ & -0.03 & $0.09^{*}$ & $0.09^{*}$ \\
\hline 4. Traditional victimisation $(t 2)$ & & & 1 & $0.35^{* *}$ & $0.26^{* * *}$ & $0.09^{*}$ & 0.03 & $0.12^{* * *}$ & 0.04 & 0.02 \\
\hline 5. Cybervictimisation $(t 2)$ & & & & 1 & $0.15^{* *}$ & 0.01 & $-0.09^{*}$ & -0.04 & 0.07 & $0.09^{*}$ \\
\hline 6. Depressive symptoms $(t 2)$ & & & & & 1 & -0.02 & -0.07 & 0.01 & $0.11^{* *}$ & $0.08^{*}$ \\
\hline 7. Distant advice & & & & & & 1 & $0.37^{* *}$ & $0.43^{* *}$ & -0.04 & -0.03 \\
\hline 8. Close support & & & & & & & 1 & $0.36^{* *}$ & -0.02 & $-0.22^{n}$ \\
\hline 9. Assertiveness & & & & & & & & 1 & -0.01 & 0.01 \\
\hline 10. Helplessness & & & & & & & & & 1 & 0.05 \\
\hline 11. Retaliation & & & & & & & & & & 1 \\
\hline
\end{tabular}

Notes: ${ }^{*} p<0.05 ;{ }^{* *} p<0.01$ 
Table 3. Results of the GLM analyses predicting depressive symptoms at $t 2\left(n_{M I}=675\right.$; $\left.n_{M 2}=667\right)$.

\begin{tabular}{|c|c|c|c|c|}
\hline & \multicolumn{4}{|c|}{ Depressive symptoms $t 2$} \\
\hline & \multicolumn{2}{|c|}{ Model 1} & \multicolumn{2}{|c|}{ Model 2} \\
\hline & $B$ & $p$ & $B$ & $p$ \\
\hline Intercept (constant) & 0.320 & 0.576 & -0.142 & 0.777 \\
\hline Depressive symptoms $(t 1)$ & & & 0.499 & 0.000 \\
\hline Age & 0.040 & 0.298 & 0.032 & 0.342 \\
\hline Gender (female) & 0.189 & 0.000 & 0.054 & 0.231 \\
\hline Traditional victimisation $(t 1)$ & 0.841 & 0.002 & 0.410 & 0.081 \\
\hline Cybervictimisation $(t 1)$ & 0.975 & 0.026 & 0.205 & 0.609 \\
\hline Traditional victimisation $(t 2)$ & 0.309 & 0.007 & 0.118 & 0.240 \\
\hline Cybervictimisation $(t 2)$ & 0.460 & 0.029 & 0.462 & 0.012 \\
\hline Distant advice & 0.014 & 0.701 & 0.012 & 0.704 \\
\hline Close support & -0.092 & 0.021 & -0.038 & 0.278 \\
\hline Assertiveness & 0.055 & 0.196 & 0.060 & 0.106 \\
\hline Helplessness & 0.092 & 0.020 & 0.064 & 0.066 \\
\hline Retaliation & 0.041 & 0.081 & 0.009 & 0.659 \\
\hline
\end{tabular}

Notes: Analyses were controlled for the situational variability of the cyberbullying scenarios.

higher level of depressive symptoms at $t 2$. In addition, the coping strategies helplessness and close support emerged as predictors of depressive symptoms at $t 2$. Close support predicted a lower level of depressive symptoms, whereas feeling helpless predicted a higher level of depressive symptoms. When including depressive symptoms at $t 1$ into model 2 , cybervictimisation at $t 2$ remained a significant predictor of depressive symptoms at $t 2$. That is, higher levels of cybervictimisation at $t 2$ predicted an increase in depressive symptoms over time.

\section{Moderating effects of coping strategies}

To test for possible moderating effects, interactions were included in the models. For the interaction analysis, variables were centred around the mean. In a first GLM analysis interaction effects between coping strategies and cybervictimisation at $t 1$ were tested. These analyses yielded no significant interaction effects.

Next, interactions with cybervictimisation at $t 2$ were calculated. The analysis yielded significant interaction effects for assertiveness and close support. To interpret the significant interaction effect, we used the procedures of Aiken and West (1991). The lines represent associations (slopes) between the independent and dependent variables for high and low levels $( \pm \mathrm{SD}$ ) of the moderator (Figures 1 and 2 ).

The GLM analysis yielded significant effects of assertiveness $\times$ cybervictimisation on depressive symptoms at $t 2$ (Wald $\chi^{2}(1,652)=4.12, B=0.68, p=0.042, \omega=0.08$ ). As can be seen in Figure 1, students with higher levels of cybervictimisation reported higher levels of depressive symptoms. This association was stronger when students recommended assertive coping strategies. When entering depressive symptoms at $t 1$ as a control variable into the model this moderating effect remained significant (Wald $\chi^{2}(1,643)=$ $10.87, B=0.96, p=0.001, \omega=0.13)$. Moreover, the interaction between close support and cybervictimisation was significant regarding changes in depressive symptoms from 


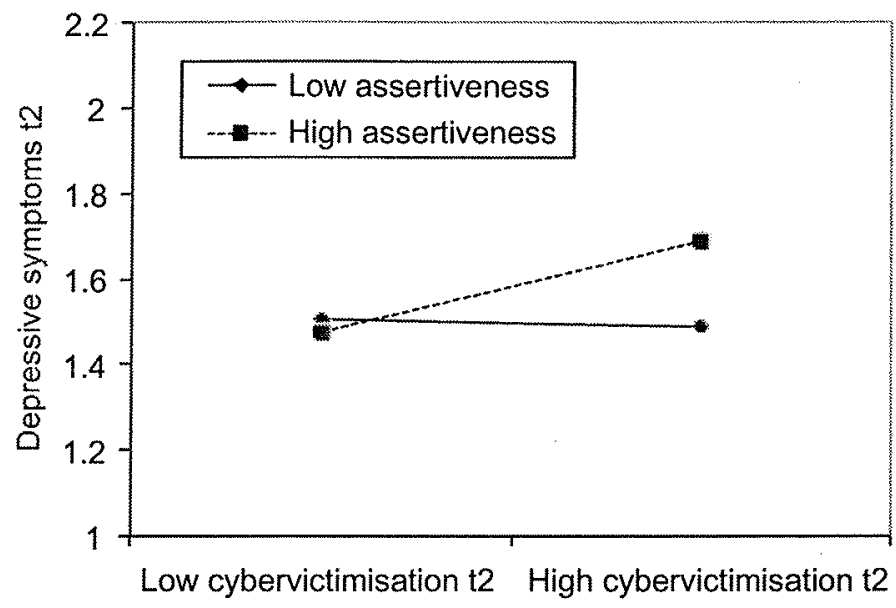

Figure 1. Interaction effect of cybervictimisation $\times$ assertiveness (on depressive symptoms at $t 2$ ).

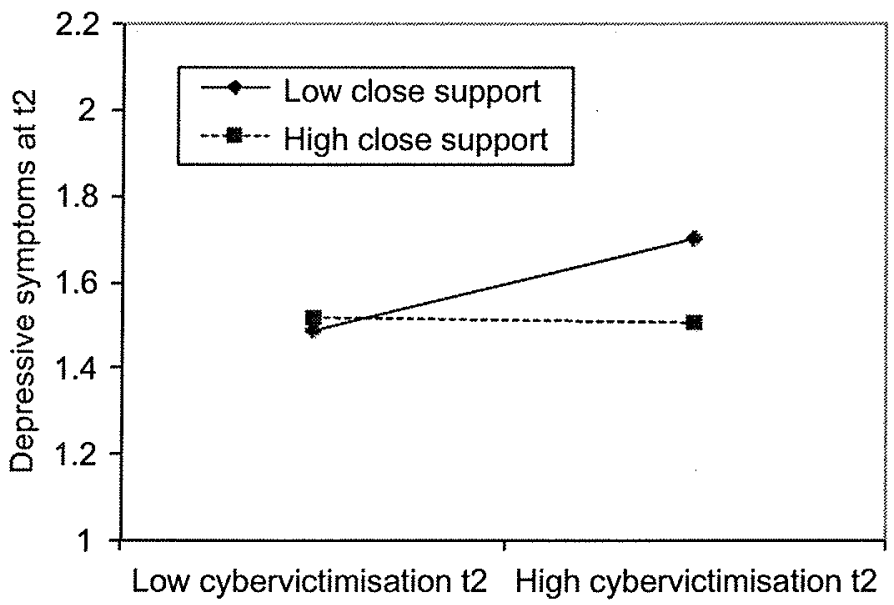

Figure 2. Interaction effect of cybervictimisation $\times$ close support (controlled for depressive symptoms at $t 1$ ).

$t 1$ to $t 2$ (Wald $\chi^{2}(1,643)=4.43, B=-0.61, p=0.035, \omega=0.08$ ). Figure 2 shows that cybervictimisation is more strongly associated with depressive symptoms at $t 2$ when students report low levels of social support, i.e. higher levels of social support seem to buffer the negative impact of cybervictimisation.

\section{Discussion}

This study examined whether cybervictimisation is a longitudinal risk factor for depressive symptoms. Furthermore, the impact of recommending specific cybervictimisation coping strategies on the association between cybervictimisation and depressive symptoms was explored. The results show that cybervictimisation is a longitudinal risk factor for adolescents' depressive symptoms above and beyond traditional victimisation. The results also suggest that social support may buffer the negative impact of cybervictimisation, whereas assertive reaction may aggravate this negative impact. 


\section{Impact of victimisation on depressive symptoms}

Results show that being victimised by traditional means and in cyberspace is a concurrent and longitudinal predictor of a higher level of depressive symptoms. These findings are in line with our expectations and with former cross-sectional studies (e.g. Baker and Tanrikulu 2010; Desjardins and Leadbeater 2011; Pouwelse et al. 2011; Ybarra 2004). The bivariate analyses also confirm that students who are victimised in one context (e.g. real world) are at a higher risk of becoming victimised in the other context, too (e.g. cyberspace). Despite this overlap, cybervictimisation emerges as an additional risk factor in regard to depressive symptoms. When analysing the effect of victimisation on changes in depressive symptoms over time, only cybervictimisation (and not traditional victimisation) at $t 2$ emerges as a significant predictor. Therefore, being victimised online could be even more strongly related to depressive symptoms than traditional forms of victimisation. An additional and stronger negative impact of cybervictimisation on depressive symptoms could be explained by the larger audience provided in cyberspace (Kowalski and Limber 2007; Smith et al. 2008; Sticca et al. in press), which might increase feelings of helplessness and shame in the victim. The finding might also be related to the fact that cybervictimisation is not confined by time and place. If adolescents are victims of traditional victimisation they can withdraw from this harassment after school. Cybervictims never can rest because at home the victimisation goes on with text messages on their mobile phone, on Facebook, and in videos on YouTube and the like.

\section{Coping strategies in cybervictimisation}

Students indicated what coping strategies a hypothetical cybervictim should use in a specific cyberbullying situation. Close support and assertiveness were the most frequently recommended coping strategies, whereas helpless reactions and retaliation were less frequently recommended.

Talking to friends and/or parents might be one of the first ideas that come to mind when thinking about what to do when experiencing cybervictimisation, since the cost associated with asking significant others for support might be comparatively low. Moreover, students emphasise the importance that significant others have the resources to help, are trustworthy and take such incidents seriously (Hinduja and Patchin 2007; Smith et al. 2008). However, it is important to note that cybervictimisation was negatively associated with close support, possibly indicating that cybervictims more seldom use close support as a coping strategy, or that they have had unsuccessful experiences. As we only asked whether they would speak to friends or parents we do not know what kind of support they would ask for: they might ask for (and receive) consolation and comfort (emotional support), or they might expect instrumental help to cope with the cybervictimisation.

In contrast to close support, the coping strategy distant advice was not significantly associated with depressive symptoms or cybervictimisation. However, traditional victimisation was positively associated with distant advice. This might indicate that students who experienced traditional victimisation may be more willing to report the incident to consultants or the police. This might, in turn, be due to their own experiences with unsuccessful attempts to stop bullying and resulting feelings of helplessness.

Another way to cope with victimisation is fighting back. In the present sample, boys recommended retaliation more often than girls, although on a lower level than other more constructive coping strategies. Gender differences often appear regarding retaliation, but it seems that the observed/reported differences are based on the way girls (e.g. more verbal) 
and boys (e.g. more physical) fight back (Hoff and Mitchell 2009). In our coping scale we used only one item addressing revenge ('I would retaliate against this person') and we did not differentiate between forms of retaliation. Therefore, we were not able to test such a hypothesis. However, our results showed that boys recommended retaliation more often than girls. Cybervictimisation $(t 2)$ was positively associated with retaliation. This, again, might indicate that victims of cybervictimisation have used such strategies. As high correlations have been reported between cybervictimisation and cyberaggression in other studies (e.g. Perren et al. 2010; Sontag et al. 2011), it is possible that some of the cybervictims are themselves rather aggressive and use this kind of reaction when facing cybervictimisation. However, we did not investigate associations between own cyberbullying behaviour and the suggested coping strategies in this study.

In line with research into avoidant coping strategies (Seiffge-Krenke and Klessinger 2000), we found that helplessness reactions (ignoring, withdrawing and self-blame) are positively associated with depressive symptoms. This finding could be explained by the general failure to use adaptive coping strategies (Asarnow, Carlson, and Guthrie 1987). Also, feelings of uncontrollability of the situation (Roth and Cohen 1986) might be more relevant in cyberbullying incidents owing to the perpetrator's greater anonymity. Holahan and colleagues (2005) suggested that the use of avoidant coping strategies leads to more acute and/or chronic life stress which, in turn, predicts higher levels of depressive symptoms.

\section{Buffering effects of coping strategies}

As hypothesised and shown in other studies, seeking support from significant others predicted lower levels of depressive symptoms, whereas avoidant and emotion-focused coping (helplessness) predicted a higher level of depressive symptoms (Ebata and Moos 1991; Hay and Meldrum 2010; Seiffge-Krenke and Klessinger 2000). In addition, higher levels of retaliation were associated with higher levels of depressive symptoms.

One of the main research questions in the present study was whether recommended coping strategies regarding a hypothetical cyberbullying scenario buffer the negative impact of cybervictimisation on depressive symptoms. The analysis showed that two coping strategies (close support and assertiveness) moderate the association between cybervictimisation and depressive symptoms. Students suggesting close support as a coping strategy showed lower levels of depressive symptoms at $t 2$. This finding is in line with the buffering hypothesis of social support (e.g. Jackson 1992) and indicates that support from significant others can reduce the negative effects of stress. However, cybervictims seem to use close support less frequently than others. That is, students recommending seeking close support are not necessarily victimised and might suggest this strategy on the basis of other situations in which they have used it successfully.

Assertiveness is commonly seen as a rather constructive and helpful way to cope with victimisation incidents (Camodeca and Goossens 2005). However, our study showed an unexpected result. For cybervictims, higher levels of assertiveness (contacting and showing own pain to the bully as well as taking technical precautions) were associated with higher levels of depressive symptoms. The moderating effect still remained when controlling for depressive symptoms at $t$. This result suggests that any contact with the perpetrator, even a constructive one, is harmful and should be avoided to reduce negative emotions. Contacting the bully, telling him that his behaviour is bad and makes one angry could lead to the outcome the perpetrator wants to get: satisfaction. Therefore, as bullying is defined by a power differential between bully and victim, contacting a bully 
might not be a useful strategy. A logical conclusion would be to avoid any contact with the bully in the hope that he loses his motivation, and eventually stops bullying. Another interpretation of this finding might be associated with aggressive victims' behaviour and difficulties: traditional bully-victims show high levels of aggressive and assertive reactions and coping behaviour (Kristensen and Smith 2003; Perren and Alsaker 2006), and also the highest level of depressive symptoms (Perren et al. 2010). Maybe traditional bully-victims are overrepresented in the group of cybervictims who recommend assertive coping strategies. Studies regarding the role of assertive coping strategies in the relationship between victimisation and emotional well-being are needed.

\section{Strengths and limitations}

The present study has a number of strengths. One is the rather large sample with a very low percentage of attrition. Furthermore, to our knowledge, this was the first study to investigate moderating effects of coping strategies on the impact of cybervictimisation on depressive symptoms using a longitudinal design. Therefore, we not only assessed perceived success, but also measured the impact of recommended coping strategies on changes in depressive symptoms. Nevertheless, the study has limitations. The ability to generalise the results is limited because we had only two time-points with a six-month interval. Moreover, coping strategies were only measured at the second assessment. Furthermore, the study relied exclusively on self-report. Therefore, the strengths of associations might be biased through shared method variance.

The coping measure also has its strengths and limitations. We asked students which coping strategies they would recommend to a hypothetical cybervictim. On the one hand, this allowed us to generate data for the whole sample and not only from cybervictims. On the other hand, we do not know whether the recommended coping strategies are actually also used by the students in similar cybervictimisation experiences. The items of our coping instrument were developed from a qualitative pilot study. We used students' answers to generate the coping items. This is certainly a strength of the instrument, as we used students' own formulations of answers and did not imply theoretical subscales that might not be relevant to their everyday experiences (Parris et al. 2011). At the same time, this procedure is a major disadvantage because we were forced to build post-hoc subscales. Although we could identify some theoretically meaningful subscales, the internal consistencies were rather low to moderate. Furthermore, we had only one item in the retaliation scale.

\section{Practical and research implications}

The implications of these findings are important for intervention programmes and evince the extent of negative impact of cybervictimisation. Being victimised in cyberspace proved to be an additional longitudinal risk factor for depressive symptoms in young adolescents. However, it remains unclear whether cybervictimisation adds a unique and qualitatively different contribution to the harm caused by traditional victimisation or whether its effect on youngsters' health is just additive and qualitatively comparable to that of traditional victimisation. Further studies should address this issue.

Close support as a coping strategy was found to be able to buffer the negative impact of this association. Analyses concerning the other coping strategies provided unexpected or no significant results. Therefore, further investigation is needed to clarify the specific longitudinal effect of different coping strategies on the association between cybervictimisation and depressive symptoms. For example, clarification is needed on whether there are 
differences of a structural and functional nature between coping mechanisms in traditional and cybervictimisation, whether coping strategies are more universal and therefore independent of context, and whether there is an overlap between the perceived and real successfulness of coping strategies. Nonetheless, the results of this study raise important questions, as well as concerns, for those young people experiencing different forms of victimisation and suffering emotional problems.

In conclusion, close support emerges as a highly adaptive coping strategy in relation to cybervictimisation. In contrast to our expectations, assertive reactions seem to aggravate the negative impact of cybervictimisation. There exists a wide range of published guidelines and recommendations to cope with cyberbullying developed by governments, schools, and private or public initiatives. However, there is a severe lack of evidence regarding the success of these suggested coping strategies (Perren et al. 2012; Smith 2012). Further investigation of successful coping strategies in relation to cybervictimisation is necessary to eliminate recommendations that may be not useful for, or may even be detrimental to, cybervictims' adaptive coping and emotional well-being.

\section{References}

Aiken, L.S., and S.G. West. 1991. Multiple regression: Testing and interpreting interactions. Newbury Park, CA: Sage.

Alsaker, F.D. 1992. Pubertal timing, overweight, and psychological adjustment. Journal of Early Adolescence 12: 396-419.

Alsaker, F.D. 2003. Quälgeister und ihre Opfer. Mobbing unter Kindern - und wie man damit umgeht. Bern: Huber Verlag.

Alsaker, F.D., and A. Brunner. 1999. Switzerland. In The nature of school bullying: A cross-national perspective, ed. P.K. Smith, Y. Morita, J. Junger-Tas, D. Olweus, R. Catalano and P. Slee, 250-63. London: Routledge.

Aricak, T., S. Siyahhan, A. Uzunhasanoglu, S. Saribeyoglu, S. Ciplak, N. Yilmaz, and C. Memmedov. 2008. Cyberbullying among Turkish adolescents. Cyberpsychology \& Behavior 11: 253-61.

Asarnow, J.R., G.A. Carlson, and D. Guthrie. 1987. Coping strategies, self-perceptions, hopelessness, and perceived family environments in depressed and suicidal children. Journal of Consulting and Clinical Psychology 55: 361-6.

Baker, O.E., and I. Tanrikulu. 2010. Psychological consequences of cyber bullying experiences among Turkish secondary school children. Procedia Social and Behavioral Sciences 2: 2771-2776.

Bhat, C.S. 2008. Cyber bullying: Overview and strategies for school counsellors, guidance officers, and all school personnel. Australian Journal of Guidance and Counselling 18: 53-66.

Camodeca, M., and F.A. Goossens. 2005. Children's opinions on effective strategies to cope with bullying: The importance of bullying role and perspective. Educational Research 47: 93-105.

Chi, C.W., and E. Frydenberg. 2009. Coping in the cyberworld: Program implementation and evaluation - a pilot project. Australian Journal of Guidance and Counselling 19: 196-215.

Cohen, S., and T.A. Wills. 1985. Stress, social support, and the buffering hypothesis. Psychological Bulletin 98: 310-57.

Davidson, L.M., and M.K. Demaray. 2007. Social support as a moderator between victimisation and internalizing-externalizing distress from bullying. School Psychology Review 36: 383-405.

Dehue, F., C. Bolman, and T. Vollink. 2008. Cyberbullying: Youngsters' experiences and parental perception. Cyberpsychology \& Behavior 11: 217-23.

Desjardins, T.L., and B.J. Leadbeater. 2011. Relational victimisation and depressive symptoms in adolescence: Moderating effects of mother, father, and peer emotional support. Journal of Youth and Adolescence 40: 531-44.

DiBasilio, A. 2008. Reducing bullying in middle school students through the use of student-leaders. Saint Xavier University, Chicago.

Dooley, J.J., P. Gradinger, D. Strohmeier, D. Cross, and C. Spiel. 2010. Cyber-Victimisation: The association between help-seeking behaviours and self-reported emotional symptoms in Australia and Austria. Australian Journal of Guidance \& Counselling 20: 194-209. 
Ebata, A.T., and R.H. Moos. 1991. Coping and adjustment in distressed and healthy adolescents. Journal of Applied Developmental Psychology 12: 33-54.

Eschenbeck, H. 2010. Bewältigung alltäglicher Stresssituationen von Kindern und Jugendlichen. Zeitschrift für Gesundheitspsychologie 18: 103-18.

Flouri, E., and A. Buchanan. 2002. Life satisfaction in teenage boys: The moderating role of father involvement and bullying. Aggressive Behavior 28: 126-33.

Gradinger, P., D. Strohmeier, and C. Spiel. 2009. Traditional bullying and cyberbullying identification of risk groups for adjustment problems. Zeitschrift für Psychologie/Journal of Psychology 217 : 205-13.

Hampel, P., S. Manhal, and T. Hayer. 2009. Direct and relational bullying among children and adolescents: Coping and psychological adjustment. School Psychology International 30: 474-90.

Hay, C., and R. Meldrum. 2010. Bullying victimisation and adolescent self-harm: Testing hypotheses from general strain theory. Journal of Youth and Adolescence 39: 446-59.

Hinduja, S., and J.W. Patchin. 2007. Offline consequences of online victimisation: School violence and delinquency. Journal of School Violence 6, no. 3: 89-112.

Hoff, D.L., and S.N. Mitchell. 2009. Cyberbullying: Causes, effects, and remedies. Journal of Educational Administration 47: 652-65.

Holahan, C.J., R.H. Moos, C.K. Holahan, P.L. Brennan, and K.K. Schutte. 2005. Stress generation, avoidance coping, and depressive symptoms: A 10-year model. Journal of Consulting and Clinical Psychology 73: 658-66.

Holsen, I., P. Kraft, and J. Vitterso. 2000. Stability in depressed mood in adolescence: Results from a 6-year longitudinal panel study. Journal of Youth and Adolescence 29: 61-78.

Holt, M.K., and D.L. Espelage. 2007. Perceived social support among bullies, victims, and bullyvictims. Journal of Youth and Adolescence 36: 984-94.

Jackson, P.B. 1992. Specifying the buffering hypothesis - Support, strain, and depression. Social Psychology Quarterly 55: 363-78.

Juvonen, J., and E.F. Gross. 2008. Extending the school grounds? Bullying experiences in cyberspace. Journal of School Health 78: 496-505.

Kelley, H.H., and J.L. Michela. 1980. Attribution theory and research. Annual Review of Psychology 31: 457-501.

Kochenderfer-Ladd, B. 2004. Peer victimisation: The role of emotions in adaptive and maladaptive coping. Social Development 13: 329-49.

Kochenderfer-Ladd, B., and K. Skinner. 2002. Children's coping strategies: Moderators of the effects of peer victimisation? Developmental Psychology 38: 267-78.

Kowalski, R.M., and S.P. Limber. 2007. Electronic bullying among middle school students. Journal of Adolescent Health 41, 6 Suppl. no. 1: S22-30.

Kowalski, R.M., S.P. Limber, and P.W. Agatston. 2008. Cyber bullying: Bullying in the digital age. Malden, MA: Blackwell.

Kristensen, S.M., and P.K. Smith. 2003. The use of coping strategies by Danish children classed as bullies, victims, bully/victims, and not involved, in response to different (hypothetical) types of bullying. Scandinavian Journal of Psychology 44: 479-88.

Lazarus, R.S. 2006. Emotions and interpersonal relationships: Toward a person-centered conceptualization of emotions and coping. Journal of Personality 74: 9-46.

Lodge, J., and S.S. Feldman. 2007. Avoidant coping as a mediator between appearance-related victimisation and self-esteem in young Australian adolescents. British Journal of Developmental Psychology 25: 633-42.

Machmutow, K., and S. Perren. 2011a. Coping with cyberbullying: Successful and unsuccessful coping strategies. Poster presented at the 12th congress of the SGP, September 13, in Fribourg, Switzerland.

Machmutow, K., and S. Perren. 2011b. Coping with cyberbullying: Successful and unsuccessful coping strategies. Poster presented at the 3rd COST workshop on cyberbullying, May 13, in Turku, Finland.

Mason, K.L. 2008. Cyberbullying: A preliminary assessment for school personnel. Psychology in the Schools 45: 323-48.

Mishna, F., M. Saini, and S. Solomon. 2009. Ongoing and online: Children and youth's perceptions of cyber bullying. Children and Youth Services Review 31: 1222-8.

Mitchell, K.J., D. Finkelhor, and J. Wolak. 2003. The exposure of youth to unwanted sexual material on the internet - A national survey of risk, impact, and prevention. Youth \& Society 34: 330-58. 
Mitchell, K.J., M. Ybarra, and D. Finkelhor, 2007. The relative importance of online victimisation in understanding depression, delinquency, and substance use. Child Maltreatment 12: $314-24$.

Parris, L., K. Varjas, J. Meyers, and H. Cutts. 2011. High school students' perceptions of coping with cyberbullying. Youth \& Society 20, no. 10: 1-23.

Patchin, J.W., and S. Hinduja. 2006. Bullies move beyond the schoolyard: A preliminary look at cyberbullying. Youth Violence and Juvenile Justice 4: 148-69.

Perren, S., and F.D. Alsaker. 2006. Social behavior and peer relationships of victims, bully-victims, and bullies in kindergarten. Journal of Child Psychology and Psychiatry 47: 45-57.

Perren, S., L. Corcoran, H. Cowie, F. Dehue, D. Garcia, C. McGuckin, A. Sevcikova, P. Tsatsou, and T. Völlink. 2012. Coping with cyberbullying: Review of evidence regarding successful strategies. Manuscript submitted for publication.

Perren, S., J. Dooley, T. Shaw, and D. Cross. 2010. Bullying in school and cyberspace: Associations with depressive symptoms in Swiss and Australian adolescents. Child and Adolescent Psychiatry and Mental Health 4, no. 28: 1-10.

Perren, S., and E. Gutzwiller-Helfenfinger. 2012. Cyberbullying and traditional bullying in adolescence: Differential roles of moral disengagement, moral emotions, and moral values. European Journal of Developmental Psychology 9: 195-209.

Pouwelse, M., C. Bolman, H. Lodewijkx, and M. Spaa. 2011. Gender differences and social support: Mediators or moderators between peer victimisation and depressive feelings? Psychology in the Schools 48: 800-14.

Price, M., and J. Dalgleish. 2010. Cyberbullying: Experiences, impacts and coping strategies as described by Australian young people. Youth Studies Australia 29, no. 2: 51-9.

Rigby, K. 2000. Effects of peer victimisation in schools and perceived social support on adolescent well-being. Journal of Adolescence 23: 57-68.

Roth, S., and L.J. Cohen. 1986. Approach, avoidance, and coping with stress. American Psychologist 41: 813-19.

Seiffge-Krenke, I, and N. Klessinger. 2000. Long-term effects of avoidant coping on adolescents' depressive symptoms. Journal of Youth and Adolescence 29: 617-30.

Singh, P., and K. Bussey. 2010. Peer victimisation and psychological maladjustment: The mediating role of coping self-efficacy. Journal of Research on Adolescence 21: 420-33.

Skinner, E.A., K. Edge, J. Altman, and H. Sherwood. 2003. Searching for the structure of coping: A review and critique of category systems for classifying ways of coping. Psychological Bulletin 129: 216-69.

Slonje, R., and P.K. Smith. 2008. Cyberbullying: Another main type of bullying? Scandinavian Journal of Psychology 49: 147-54.

Smith, P.K. 2012. Cyberbullying and cyber aggression. In Handbook of school violence and school safety: International research and practice, ed. S.R. Jimerson, A.B. Nickerson, M.J. Mayer and M.J. Furlong, 93-103. New York: Routledge.

Smith, P.K., J. Mahdavi, M. Carvalho, S. Fisher, S. Russell, and N. Tippett. 2008. Cyberbullying: Its nature and impact in secondary school pupils. Journal of Child Psychology and Psychiatry 49: $376-85$.

Smith, P.K., and R. Slonje. 2010. Cyberbullying: The nature and extent of a new kind of bullying, in and out of school. In Handbook of bullying in schools: An international perspective, ed. S.R. Jimerson, S.M. Swearer and D.L. Espelage, 249-62. New York: Routledge.

Sontag, L.M., K.H. Clemans, J.A. Graber, and S.T. Lyndon. 2011. Traditional and cyber aggressors and victims: A comparison of psychosocial characteristics. Journal of Youth and Adolescence 40: 392-404.

Spears, B., P. Slee, L. Owens, and B. Johnson. 2009. Behind the scenes and screens insights into the human dimension of covert and cyberbullying. Zeitschrift für Psychologie/Journal of Psychology 217: $189-96$.

Stacey, E. 2009. Research into cyberbullying: Student perspectives on cybersafe learning environments. Informatics in Education 8: 115-30.

Sticca, F., S. Ruggieri, F.D. Alsaker, and S. Perren. In press. Longitudinal risk factors for cyberbullying in adolescence. Journal of Community \& Applied Social Psychology.

Sweeting, H., R. Young, P. West, and G. Der. 2006. Peer victimisation and depression in early-mid adolescence: A longitudinal study. British Journal of Educational Psychology 76: $577-94$. 
Tenenbaum, L.S., K. Varjas, J. Meyers, and L. Parris. 2011. Coping strategies and perceived effectiveness in fourth through eighth grade victims of bullying. School Psychology International 32 : 263-87.

Tokunaga, R.S. 2010. Following you home from school: A critical review and synthesis of research on cyberbullying victimisation. Computers in Human Behavior 26: 277-87.

Topcu, C., O. Erdur-Baker, and Y. Capa-Aydin. 2008. Examination of cyberbullying experiences among Turkish students from different school types. Cyberpsychology \& Behavior 11: 643-8.

Wang, J., T.R. Nansel, and R.J. Jannotti. 2011. Cyber and traditional bullying: Differential association with depression. Journal of Adolescent Health 48: 415-7.

Yamasaki, K., and K. Uchida. 2006. Relation of positive effect with emotion-focused coping in Japanese undergraduates. Psychological Reports 98: 611-20.

Ybarra, M.L. 2004. Linkages between depressive symptomatology and internet harassment among young regular internet users. Cyberpsychology \& Behavior 7: 247-57. 